\title{
Study on the Relations and Differences of Differential Mean Value Theorems
}

\author{
Xin Wang, Shiqin Wang, Cheng Wang* \\ College of Mathematics and Computer Science, Huanggang Normal University, \\ Huanggang 438000, China \\ ${ }^{*}$ Corresponding Author: wangc80@163.com
}

\section{Keywords: Roll's theorem; Lagrange's theorem; Cauchy's theorem}

Abstract. The differential mean value theorem plays an important role in the differential calculus. In this paper, by comparing and analyzing the premise conditions and conclusions of three differential mean value theorems, we discuss the similarities and differences between them at first. Then, according to different principles, we summarize two kinds of typical methods of proving Lagrange's theorem and Cauchy's theorem which will be helpful for readers to understand the differential mean value theorems.

\section{Introduction}

Mathematics is an independent discipline with some basic elements such as the logic and intuition, analysis and reasoning, and commonness and individuality. As a normal member of mathematics, the differential calculus is not an exception of the norm. The natures of functions are closely related to their derivatives, especially the first order derivative which describes the changing rate of the independent variable. It is easy to find that the differential mean value theorem precisely discussed the relationship between the function and its first order derivative.

In terms of the process of the proof of the theorems, the approach most literatures adopt is that they firstly demonstrated Roll's theorem by means of Fermat's theorem, second, they, saw the Roll's constructed theorem as the foundation, constructed auxiliary function which meet the basic conditions of the theorem to prove Lagrange mean value theorem and Cauchy mean value theorem. The typical examples are reference [1] and [2] in which the Roll's theorem's proving process and ideas are simple and clear while the other two theorems' are not. The readers may think that their proving processes are brief at the fist glance, while when they speculate how the auxiliary functions were constructed, they won't keep this point any more. There are also a lot of references put forward their own opinions on the proof of the three differential theorems and list respective theoretical evidences, but mostly are not comprehensive enough. In order to help the readers understand the proof of the Lagrange theorem and Cauchy theorem more comprehensive, this paper mainly provide two methods of them.

\section{The Similarities and Differences of Three Differential Mean Value Theorems}

For the convenience of the description and the proof of the following, we introduced the concrete contents of the three theorems at first.

Theorem 1 [3] (Roll's theorem) If a real-valued function $f$ satisfies the following conditions: (a) continuous on the closed interval $[a, b]$; (b) differentiable in the open interval $(a, b)$; (c) $f(a)=f(b)$, then there exists at least one point $\xi$ in open interval $(a, b)$ such that $f^{\prime}(\xi)=0$.

Theorem 2 [3] (Lagrange's theorem) If a real-valued function $f$ satisfies the following conditions: (a) continuous on the closed interval $[a, b]$; (b) differentiable in the open interval $(a, b)$; then there exists at least one point $\xi$ in open interval $(a, b)$ such that $f^{\prime}(\xi)=\frac{f(b)-f(a)}{b-a}$. 
Theorem 3[3] (Cauchy's theorem) If two real-valued functions $f$ and $g$ both meet the following conditions: (a) continuous on the closed interval $[a, b]$; (b) differentiable in the open interval $(a, b)$; (c) not equal to zero at the same time; (d) $g(a) \neq g(b)$, then there exists a point $\xi \in(a, b)$ such that $\frac{f^{\prime}(\xi)}{g^{\prime}(\xi)}=\frac{f(b)-f(a)}{g(b)-g(a)}$.

Comparing the preconditions of the three theorems, it is easy to find that the first two conditions are "(a) continuous in a closed interval; (b) differentiable in the open interval". Because of the closed interval is limited, putting the condition (a) in the first place indicated that the three theorems are discussed in limited interval. Function differentiable is sufficient but unnecessary condition for continuous function, therefore, seemingly, the condition 'differentiable in the open interval' in (b) partly duplicates the "continuous" emphasized in (a), in fact, condition (a) emphasizes the scope of the function. Changing the open interval in (b) into the closed interval the two conditions will be severer; this leads to narrow the scope of the conclusion. If change 'the closed interval' in (a) in to 'the open interval' the condition will be weakened, accordingly, the scope of the conclusion is broadened. In order to expand its scope of application and ensure its accuracy, it should meet the conditions (a) and (b) simultaneously.

\section{Discussing the Relation Between the Theorems}

Many ways can be used to prove the differential theorem of mean value. The following will prove the Lagrange's theorem and Cauchy's theorem by constructing new auxiliary function and rotating coordinate. It is not difficult to find that the way to proving those two theorems are similar, but there exist differences in detail.

According to the comparison and analysis, we can find that references [1] and [2] are using Fermat's theorem to prove Roll's Theorem, then constructed proper auxiliary function to prove Lagrange's theorem and Cauchy's theorem on the base of Roll's Theorem. This method is very common in solving other problems, but we can hardly know how the function constructed which leads to difficult. By the prompt of [2], we can use the area of the triangle to construct the auxiliary function, and the difference of the auxiliary function and between another function in reference [4] is a constant.

The proof of Lagrange's theorem: constructing auxiliary functions $F(x)=\left|\begin{array}{lll}f(a) & a & 1 \\ f(b) & b & 1 \\ f(x) & x & 1\end{array}\right|$, then $F(x)=(a-b) f(x)-[f(a)-f(b)] x+[b f(a)-a f(b)]$. Since $f(x)$ continuous on $[a, b]$ and differentiable in $(a, b), F(x)$ continuous on $[a, b]$ and differentiable in $(a, b)$, then we can take the derivative of $F(x): F^{\prime}(x)=(a-b) f^{\prime}(x)-[f(a)-f(b)]$. Obviously, $F(a)=F(b)=0$.

To sum up, $F(x)$ meet the condition of Roll's Theorem, so there exist at least a point $\xi$, that is $F^{\prime}(\xi)=0$, then $F^{\prime}(\xi)=(a-b) f^{\prime}(\xi)-[f(a)-f(b)]=0$. Finally, the equation of Lagrange's theorem is obtained: $f^{\prime}(\xi)=\frac{f(b)-f(a)}{b-a}$.

The proof above simplified the function which constructed by geometrical significance, so as to obtain a new auxiliary function. The following method of proving the Cauchy's theorem is the same to above one.

The process of proving the structure of the auxiliary function in reference [5] is the same in this paper, but it takes the derivative to constructed determinant directly, this method requires to the use of a less common lemma. But in this paper, we convert the determinant to function, then taking the derivative to the function; therefore, the calculation process is simple and easy to understand.

Whether it is constructing analytical expressions or determining auxiliary function based on the geometric meaning of the determinant, they all will draw corresponding conclusions through algebraic operation. Similarly, in reference [6], the author constructed auxiliary functions by using coordinate rotation 
transformation and proved the Lagrange's theorem through algebraic operation. This method provides a simple idea of proof which is worth learning. In the following, by means of coordinate rotation transformation, we will put the function which can reflect the theorems into the coordinate system and draw the same conclusion according to its geometric significance.

The proof of Lagrange's theorem: set a real-valued function $f$ is continuous on the closed interval $[a, b]$, differentiable in the open interval $(a, b)$.First, introducing the rotating coordinate transformation: $\left\{\begin{array}{l}x=X \cos \theta-Y \sin \theta \\ y=X \sin \theta+Y \cos \theta\end{array}\right.$.

Since $D=\left|\begin{array}{rr}\cos \theta & -\sin \theta \\ \sin \theta & \cos \theta\end{array}\right|=1 \neq 0$, then we get $\left\{\begin{array}{l}X(x)=x \cos \theta+y \sin \theta \\ Y(x)=-x \sin \theta+y \cos \theta\end{array}\right.$. Maintaining the origin's original position and rotating coordinate system $x o y$, we will get a new coordinate system in which the $x$ axis is parallel to line which is remarked as $l$ (the tangent of $f(x)$ at the point of $x=\xi$ ) and we can have $k_{l}=\frac{f(b)-f(a)}{b-a}$. Since $l / / x$, then we have

$$
k_{l}=k_{x^{\prime}}=\tan \theta=\frac{f(b)-f(a)}{b-a}
$$

That is to say, the rotation angle $\theta=\arctan \frac{f(b)-f(a)}{b-a}$. Obviously, the function $Y(x)=-x \sin \theta+f(x) \cos \theta$ is continuous on the closed interval $[a, b]$ and differentiable in the open interval $(a, b)$. According to (1), we can get that $-a \sin \theta+f(a) \cos \theta=-b \sin \theta+f(b) \cos \theta$, then it is easy to get $Y(a)=Y(b)$. To sum up, the function $Y(x)$ meet the conditions (a), (b) and (c) of Roll's theorem. Therefore, there exists at least one point $\xi \in(a, b)$ such that $f^{\prime}(\xi)=0$, that is to say $Y^{\prime}(\xi)=-\sin \theta+f^{\prime}(\xi) \cos \theta=0$, so we can get $f^{\prime}(\xi)=\tan \theta=\frac{f(b)-f(a)}{b-a}$.

The method using to prove Cauchy's theorem in the following is similar to the method using coordinate rotating to prove Lagrange's theorem above except for some difference on the details.

The proof of Cauchy's theorem: constructing parameter function

$$
\left\{\begin{array}{l}
x=g(t) \\
y=f(t)
\end{array} a \leq t \leq b\right.
$$

On the basis of the conditions to the theorem, it is easy to know that the image of (2) is a continuous and smooth curve in the xoy plane whose two endpoints are remarked as $A(g(a), f(a))$ and $B(g(b), f(b))$. As is shown in the figure 2, remarking the intersection angle between $x$ axis and the line $l$ which includes two points $A$ and $B$ as $\alpha$, the length of line segment $A B$ as $r$,maintaining the origin's original position and rotating coordinate system $x o y$, we will get a new coordinate system in which the $x$ ' axis is parallel to line $l$ and the projection interval original curve is $\left[g^{\prime}(a), g^{\prime}(b)\right]$. Similarly, by means of rotating coordinate transformation, We can get the parameter equation of the original curve in the new coordinate system as following:

$$
\left\{\begin{array}{l}
g^{\prime}(t)=g(t) \frac{g(b)-g(a)}{r}+f(t) \frac{f(b)-f(a)}{r} \\
f^{\prime}(t)=-g(t) \frac{f(b)-f(a)}{r}+f(t) \frac{g(b)-g(a)}{r}
\end{array} .\right.
$$


According to the conditions of Cauchy's theorem, setting $\frac{d g^{\prime}}{d t} \neq 0$, it is easy to prove that the equation above satisfies the conditions of Roll's theorem. Then there exists a $\xi \in(a, b)$, such that $g^{\prime}(\xi) \in\left(g^{\prime}(a), g^{\prime}(b)\right)$ and $\frac{d f^{\prime}}{d g^{\prime}}=0\left(g^{\prime}=g^{\prime}(\xi)\right)$. That is to say,

$$
\frac{d f^{\prime}}{d g^{\prime}}=\frac{-g^{\prime}(t) \frac{f(b)-f(a)}{r}+f^{\prime}(t) \frac{g(b)-g(a)}{r}}{g^{\prime}(t) \frac{g(b)-g(a)}{r}+f^{\prime}(t) \frac{f(b)-f(a)}{r}}=0\left(g^{\prime}=g^{\prime}(\xi)\right) .
$$

Simplifying the equation, we can get $\frac{f^{\prime}(\xi)}{g^{\prime}(\xi)}=\frac{f(b)-f(a)}{g(b)-g(a)}$ (among which $\xi \in(a, b)$ ).

\section{Conclusions}

In many literatures, such as [6] and [7], the authors introduced different methods to prove three differential mean value theorems, and each method uses the theorem which is familiar to us as a tool to prove these three theorems. But few of them can find the connections between the three theorems in the process of proving. In this paper, we first analyze the relation between the three theorems, and prove their inner relation, so that we can understand the relation between the function and the first order derivative deeper.

\section{Acknowledgments}

This work is supported by the Reform Project of Transformation Development of Huanggang Normal University (No. xfg2015C19).

\section{References}

[1] Mathematics Department of East China Normal University, Mathematical Analysis, Higher Education Press, Beijing, 2001.

[2] J.X. Chen, Mathematical analysis, Higher Education Press, Beijing, 2005.

[3] K. Baron, A proof of the Cauchy Theorem, University of Science 18 (2010) 81-82.

[4] G.W. Yang, Proof of differential mean value theorem, Journal of Luoyang University 12 (2006) 49-52.

[5] Z.H. Liu, The proof of the Lagrange theorem of the value, Journal of Tianjin University of Commerce 5 (2002) 35-36.

[6] D.L. Huang, The five methods are used to prove Cauchy's Theorem, Journal of Huzhou Teachers College 6 (2003) 27-31.

[7] C.X. Zhang, The application of interval sets theorem to prove the theorem of mean value, Journal of Harbin University of Commerce 12 (2005) 794-796. 